\title{
Chromospheric jets around the edges of sunspots ${ }^{\star}$
}

\author{
R. J. Morton \\ Solar Physics and Space Plasma Research Centre (SP $\left.{ }^{2} \mathrm{RC}\right)$, University of Sheffield, Hicks Building, Hounsfield Road, \\ Sheffield S3 7RH, UK \\ e-mail: r.j.morton@sheffield.ac.uk
}

Received 29 February 2012 / Accepted 3 May 2012

\begin{abstract}
Aims. Evidence is beginning to be put forward that demonstrates the role of the chromosphere in supplying energy and mass to the corona. We aim to assess the role of chromospheric jets in active region dynamics.

Methods. Using a combination of the Hinode/SOT Ca II H and TRACE $1550 \AA$ and $1600 \AA$ filters we examine chromospheric jets situated at the edge of a sunspot.

Results. Analysis reveals a near continuous series of jets, that raise chromospheric material into the low corona above a sunspot. The jets have average rise speeds of $30 \mathrm{~km} \mathrm{~s}^{-1}$ and a range of $10-100 \mathrm{~km} \mathrm{~s}^{-1}$. Enhanced emission observed at the jets leading edge suggests the formation of a shock front. Increased emission in TRACE bandpasses above the sunspot and the disappearance of the jets from the Ca II filter suggests that some of the chromospheric jet material is at least heated to $\sim 0.1 \mathrm{MK}$. The evidence suggests that the jets could be a source of steady, low-level heating for active region features.
\end{abstract}

Key words. magnetohydrodynamics (MHD) - plasmas - shock waves - Sun: chromosphere - sunspots

\section{Introduction}

Dynamic jets of plasma observed in the solar chromosphere are currently the subject of much interest. De Pontieu et al. (2011) has demonstrated the association between spicules and material heated to EUV temperatures ( $T \sim 0.2-1.0 \mathrm{MK})$, suggesting they play an important role in supplying heated plasma to maintain the quiet corona. Madjarska et al. (2011) provides further evidence for a connection between coronal hole spicules and transition region emission but find no corresponding EUV $(T>0.3 \mathrm{MK})$ emission. The importance of plasma jets in atmospheric dynamics is also highlighted in observations of large solar jets, which show how multiple, small jets can launch large quantities of cool (chromospheric) and heated plasma into the corona (e.g., Moore et al. 2011; Scullion et al. 2011; Morton et al. 2012b).

Further to this, recent observations have shown that these dynamic jets support ubiquitous transverse waves, both at the limb in spicules (De Pontieu et al. 2007c; Zaqarashvili \& Erdélyi 2009), on-disk in mottles (Kuridze et al. 2012), plumes (Tian et al. 2011b) and in larger jet structures (Cirtain et al. 2007; Liu et al. 2009; Vasheghani Farahani et al. 2009; Morton et al. 2012a). The transverse waves in spicules appear to have sufficient energy to power the solar wind and possibly heat the quiet solar corona.

The behavior and appearance of chromospheric jets is well documented for the quiet Sun, the plage of active regions and coronal holes. De Pontieu et al. (2007b) give evidence for the existence of two types of spicule, namely Type I and Type II. Type I's are characterised as jets that rise with speeds of $10-40 \mathrm{~km} \mathrm{~s}^{-1}$ and exhibit parabolic profiles in time-distance plots, indicating a portion of the jet's mass that is ejected returns

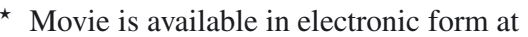
http: //www . aanda.org
}

to the surface. On the other hand, type II's are shown to accelerate while rising, reaching speeds of $50-100 \mathrm{~km} \mathrm{~s}^{-1}$ and fade from the filter without being seen to return to the surface. It is these events, so far, that have been observed to have a coronal counterpart (De Pontieu et al. 2011). The presence of a fast moving jets has also been reported on disk (Rouppe van der Voort et al. 2009).

Although it is well known that spicule-like features occur in active regions (e.g., plage regions - dynamic fibrils), details of their behavior at the foot points of coronal structures are scarce. Due to the substantial differences between active region and quiet Sun magnetic structures, it might be expected that there is a difference between the jet phenomena that inhabit the two regions. This idea is supported by observed differences between quiet Sun/coronal hole spicules (De Pontieu et al. 2007b) and dynamic fibrils in plage regions (De Pontieu et al. 2003; Tziotziou et al. 2004; De Pontieu et al. 2007a). We note that small-scale jets (widths $\sim 400 \mathrm{~km}$ ) have been identified in the chromospheric penumbra of sunspots (Katsukawa et al. 2007), however, these jets are formed along low-lying magnetic structures that probably do not penetrate into the corona.

Closely related to the subject of jets are up-flows and down flows. Flows have been observed above active regions in spectral lines corresponding to transition region (TR) temperatures. Kjeldseth-Moe et al. (1988) reported the presence of 5-20 km s${ }^{-1}$ up-flows and $40-80 \mathrm{~km} \mathrm{~s}^{-1}$ down flows in C IV (1548 $\AA$ ), which has a formation temperature of $\sim 0.1 \mathrm{MK}$. More recently, Teriaca et al. (2008) used the SUMER (Solar Ultraviolet Measurements of Emitted Radiation) instrument to investigate the TR above sunspots, finding pervasive down flows on the order of $10-30 \mathrm{~km} \mathrm{~s}^{-1}$ in Si IV (1403 $\left.\AA, T \sim 0.073 \mathrm{MK}\right)$. A number of other studies have also shown that down flows are associated with higher TR temperatures $(T \sim 0.6-1.0 \mathrm{MK})$ 
and high speed up-flows are seen at coronal temperatures $(T>$ 1 MK), e.g. Marsch et al. (2004, 2008), Bryans et al. (2010), Ugarte-Urra \& Warren (2011), Warren et al. (2011), Kamio et al. (2011).

On the nature of the TR above sunspots, study of Hydrogen Lyman line profiles with SUMER by Tian et al. (2009) revealed an interesting insight. The authors suggested that the TR above a sunspot is higher and more extended than the TR in plage regions. They also conjectured there might be less chromospheric plasma above sunspots.

We aim to examine further the role that chromospheric phenomenon play in the dynamics of active region structures connected with sunspots. We investigate the properties of chromospheric jets that surround a sunspot seen at the limb using Hinode and the Transition Region And Coronal Explorer (TRACE). Further, we demonstrate that the TR is higher and more extended above the sunspot than in the plage, as suggested by Tian et al. (2009). The chromosphere is also extended and higher above the sunspot, suggesting it is less dense in comparison to the plage chromosphere. Although it is challenging to study the chromosphere/transition region above the sunspots, imaging observations such as this one here provide an insight into the role of the chromosphere in maintaining overlying active region features.

\section{Data analysis}

The data were obtained by Hinode Solar Optical Telescope (SOT - Suematsu et al. 2008) at 01:11:00 UT 21 November 2006 till 02:00:00 UT on the same day. Hinode/SOT viewed a region on the west limb with the $\mathrm{Ca}$ II $\mathrm{H}$ broadband filter, which has a pixel size of $0.054^{\prime \prime}$ but is diffraction limited to $0.2^{\prime \prime}(145 \mathrm{~km})$. The cadence of the data is $\sim 8 \mathrm{~s}$. We performed the usual processing routine for SOT data sets with $f g \_$prep.pro. However, the data still possess a significant drift over time. We correct for this by tracking the limb over the time series and removing the trend. All images (except those shown in Fig. 1) are then rotated so that the limb faces north.

In the time series, Hinode was following the active region 10923. Hinode tracked its progress across the disk and the active region has been the subject of many investigations (e.g., Katsukawa et al. 2007). In the data series used here, the sunspot is about to cross the limb (Fig. 1) allowing a novel side-on view of the sunspots structure. The images clearly show vertical and inclined magnetic fields emanating from the region into the lower corona. A snapshot from XRT (Kano et al. 2008) shows that hot coronal plasma exists above the sunspot. We do not use the XRT data for analysis because the telescope was operating with a low cadence $(3.5 \mathrm{~min})$ at the time of interest. This cadence is much too low to observe dynamic behavior on the timescales observed in $\mathrm{Ca}$ II $\mathrm{H}$.

To emphasise magnetic structures and moving features we employ a number of standard analysis techniques. The first is unsharp masking, where, for each time frame, we subtract an image smoothed with a boxcar function of width 10 by 10 pixels from the original image. The result is seen in, e.g., Fig. 2. Second, we create running difference images of the original data by subtracting the preceding time-frame from each image.

We also use data from the $1550 \AA$ and $1600 \AA$ filters on board TRACE (Handy et al. 1999) taken on the same day. The data is subject to typical TRACE preparation routines. The data for these bandpasses is sporadic in time and only covers certain parts of the Hinode time series. Both these filters have significant contributions from the continuum and from C IV
(Handy et al. 1998) that has a formation temperature of $T \sim$ $0.1 \mathrm{MK}$. We do not use the technique developed in Handy et al. (1998) for separating the continuum emission from C IV emission as this is only suitable for intense solar features. For analysis between TRACE and Hinode/SOT images, we rebin the SOT data so that pixels correspond to TRACE resolution and we align the images via cross-correlation.

Further, data from the SOlar and Heliospheric Observatory (SOHO) Extreme ultraviolet Imaging Telescope (EIT Delaboudinière et al. 1995) is also used here and images are subject to the standard $\mathrm{SOHO} / \mathrm{EIT}$ preparation routines. The resolution of EIT is $2.56^{\prime \prime}(\sim 1000 \mathrm{~km})$, which is much too coarse to study the fine-scale dynamics, so the EIT images will only provide context for the Hinode/SOT and TRACE images.

\section{Observational features}

On viewing the unsharp masked movie of the data, it is seen that the plasma at the edge of the sunspot displays highly dynamic behavior (movie 1). Jets appear almost continuously from the chromosphere and rise into the corona up to heights of 10-15 Mm (10-20 arcsec). The jets travel along fine-scale structures with widths on the order of the diffraction limited resolution, i.e. $\sim 150-200 \mathrm{~km}$. The widths given are the values of the full-width half-maximums obtained from a Gaussian fit to the structures cross-sectional intensity profile.

Throughout the time series the plasma jets outline the magnetic field revealing that it branches out almost symmetrically above the sunspot. However, the jet features do not display the same behavior across the sunspot. It can be observed that the left and right hand sides of the sunspot display much greater activity than at the center. Images from SOHO/EIT (Fig. 1) reveal a "butterfly" shaped active region emanating from the sunspot, consisting of two sets of coronal loops highly inclined to the vertical. The brightest emission at coronal heights in all EIT channels is associated with the two sets of loops. The emission over the center of the sunspot is fainter. The overall picture available from Hinode and SOHO would suggest that the greater jet activity is associated with the coronal loops.

The left-hand side of the sunspot shows near continuous jets, with plasma emission reaching up to $15 \mathrm{Mm}$. The continuous nature of the up-flow suggests a large amount of plasma is ejected into the atmosphere but evidence for it returning to the surface is far less obvious. Occasionally, receding jets can be observed but they appear faint in intensity compared to the rising material. Continuous up-flows are also seen on the right hand side of the sunspot. However, intermittent, large plasma ejections dominate the behavior of the jets, sending them higher into the atmosphere with greater speeds (e.g., Fig. 3).

The jets can also be observed to be generated in groups spanning $\sim 2000 \mathrm{~km}$ and consist of a number of the resolution size structures (Fig. 2). These groups of jets make up the majority of the observed up-flow on the left hand side of the sunspot. To the best of our knowledge, no such collective behavior has been reported before in spicules/fibrils/mottles either in the quiet or active Sun.

Next, we determine the rise speeds of the observed jets. We place a cross-cut aligned with the jets upward trajectory (see Fig. 1) and produce time-distance diagrams from the running difference movies of the data. Figure 3 shows typical time-distance plots where we have marked on certain jet's velocities. On calculating the velocities we have ignored projection effects as we are unable to obtain any measurement of the inclination along the line of sight. Note, a number of vertical lines appear in the 
R. J. Morton: Chromospheric jets
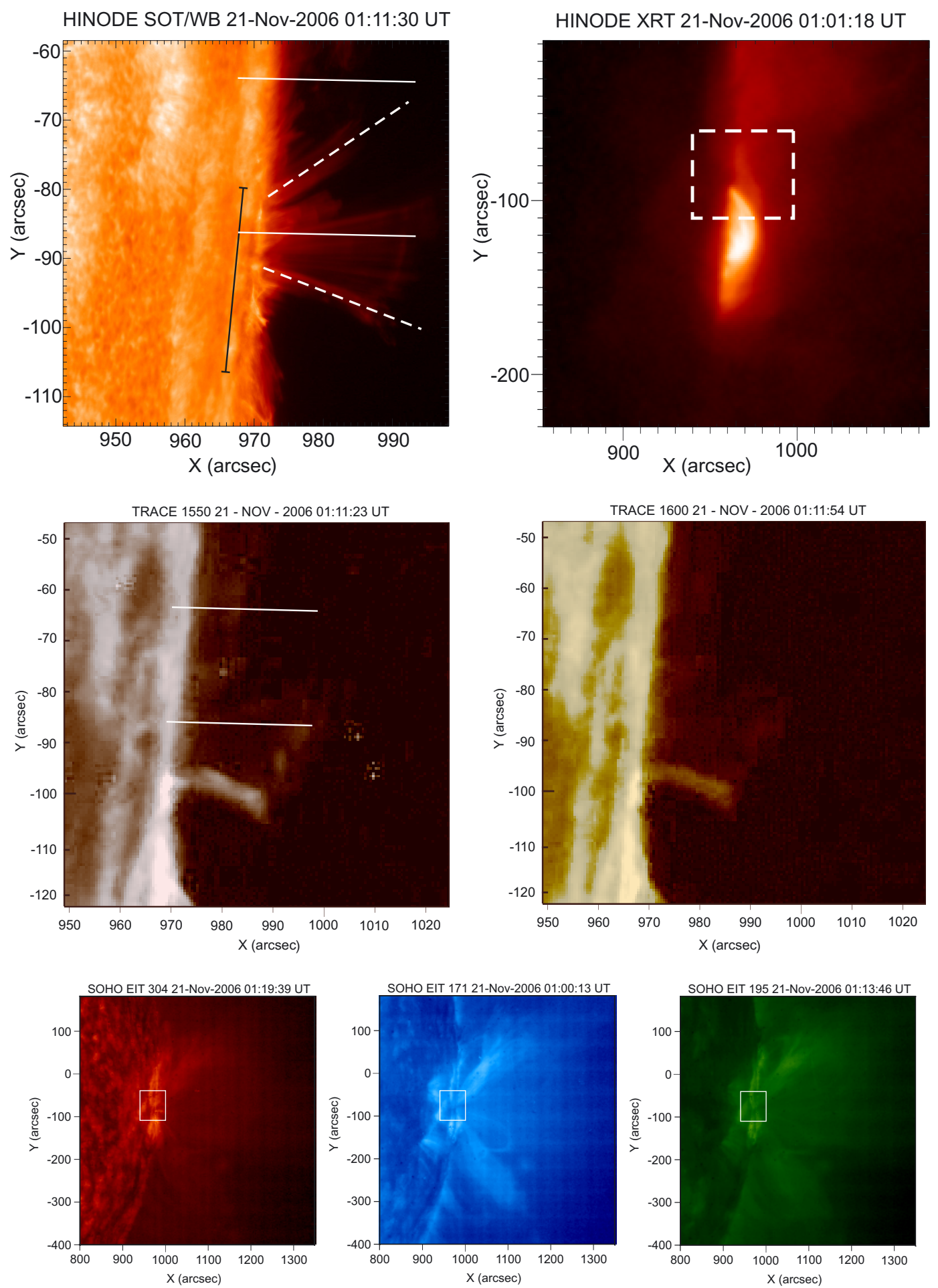

Fig. 1. The field of view as observed by Hinode, TRACE and SOHO EIT. The top left hand image shows the complete field of view for SOT. The black line shows the position of the sunspot umbra. Magnetic field lines, almost perpendicular to the surface, emanate from the sunspot boundary and are outlined by the Ca II emission. The two dashed lines mark the positions of the cross-cuts used in Fig. 3. The two solid lines mark the position of cross-cuts used in Fig. 5. The top right image shows a sub region of the XRT field of view. The white box marks the SOT field of view. The middle row shows images of the sunspot at the limb as seen by TRACE $1550 \AA$ (left) and $1600 \AA$ (right). The two solid lines in the $1550 \AA$ image mark the position of cross-cuts used in Fig. 5. The bottom row shows the corresponding SOHO/EIT $304 \AA$, $171 \AA$ and $195 \AA$ images. The solid white boxes in each image mark the SOT field of view.

time-distance plots. These appear to be the result of jets moving transversally across the sunspot, hence passing through the cross-cut. We do not identify any line in the time-distance plots as a jet that shows emission over a length of $1 \mathrm{Mm}$ in one time frame. This means we may also rule out some short lived, fast-moving ( $\left.>125 \mathrm{~km} \mathrm{~s}^{-1}\right)$ jets. Such jets should be visible on viewing the unsharp-masked images, although we can identify none.

The velocities of over one hundred jets are measured and a histogram of their velocity distribution is shown in Fig. 4. The 

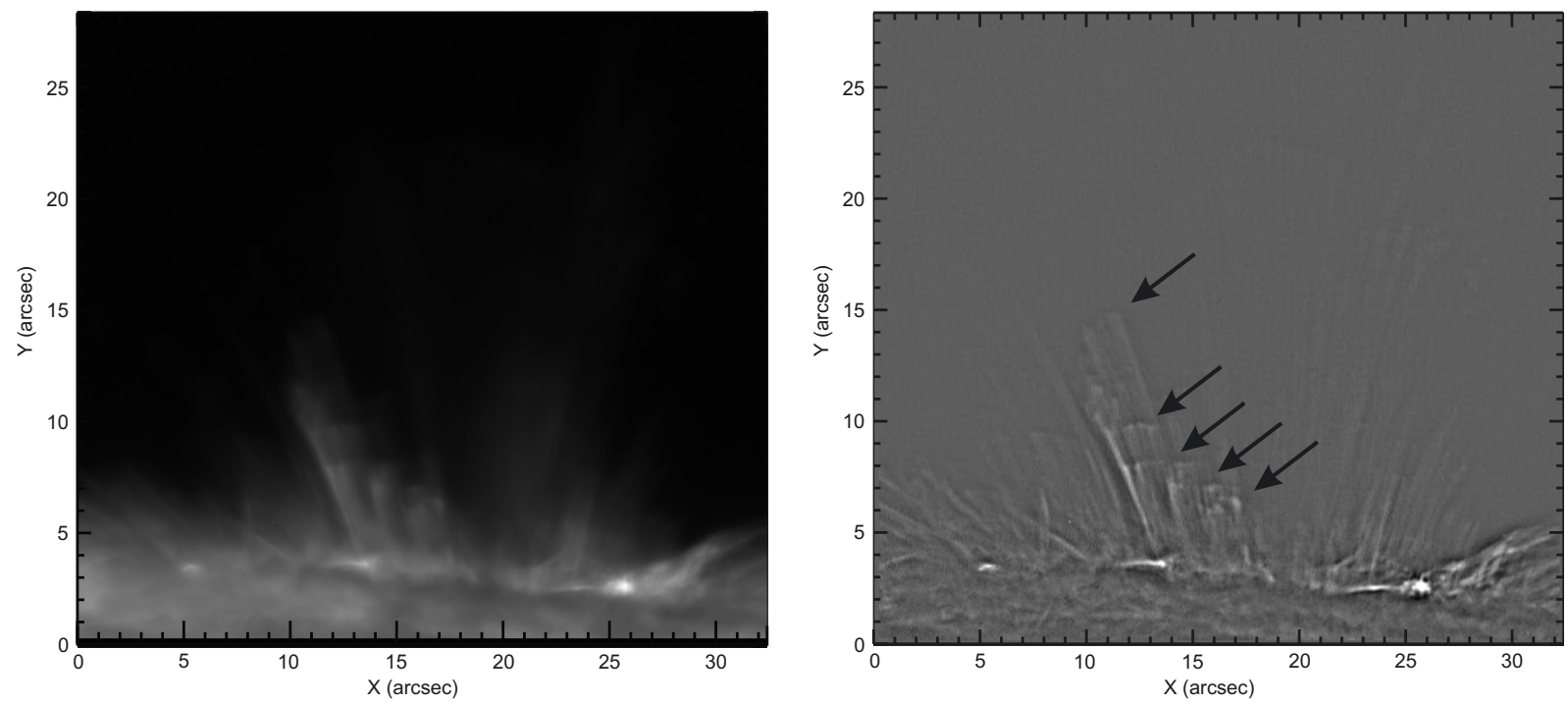

Fig. 2. A Ca II H image (left) and an unsharp masked version of the image (right). The magnetic field lines surrounding the sunspot are highlighted by up-flowing plasma jets. The jets are launched in groups along a number of field lines. These discrete groups are highlighted by the arrows. The temporal evolution can be seen in the movie available in the online addition.

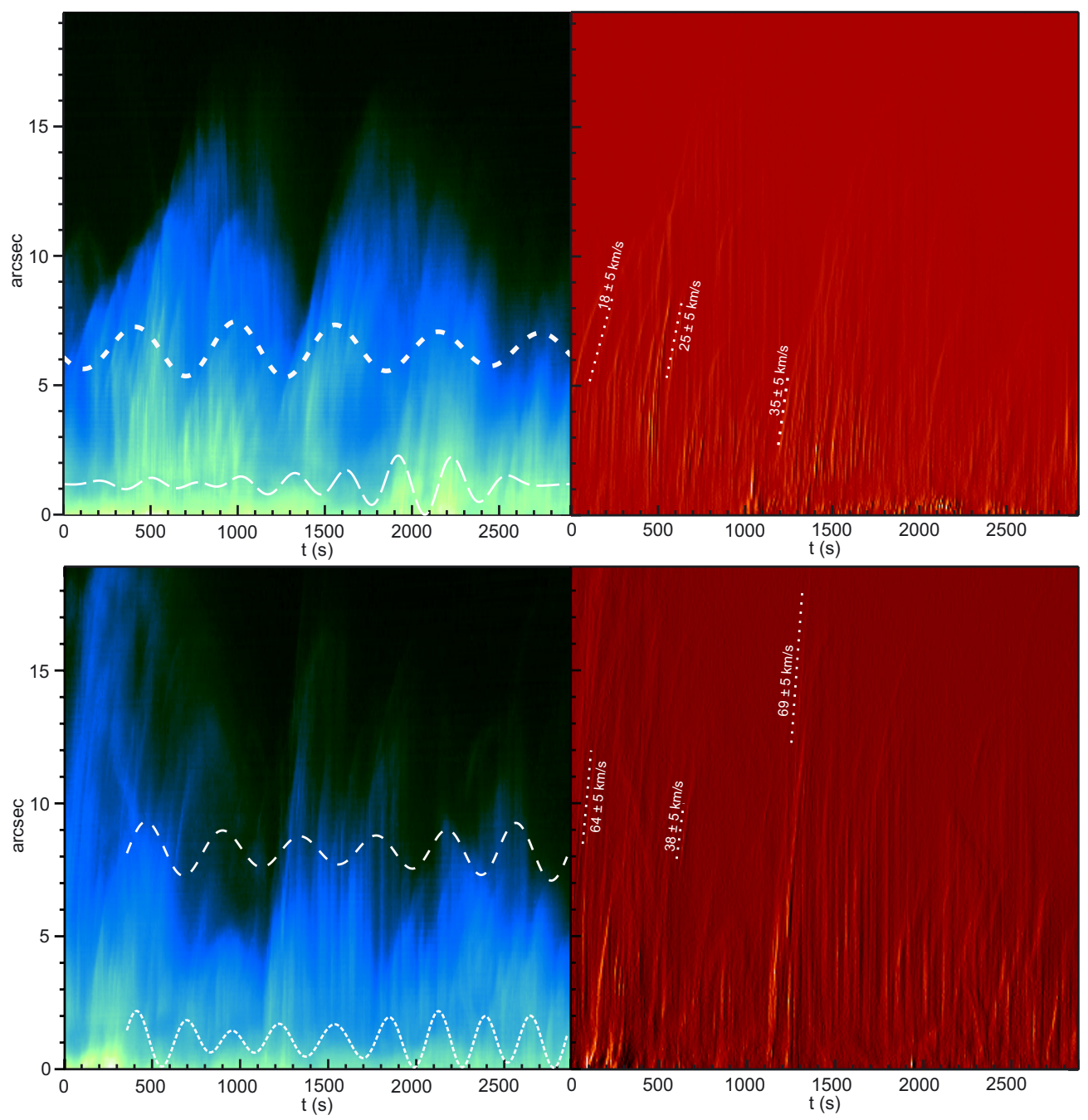

Fig. 3. The left hand column shows time-distance diagrams take at the left (upper) and right (lower) sides of the sunspot. The wavelet scales displaying the greatest power are over plotted, with the zero crossings marking the height at which the wavelet was taken. The right hand columns displays the time-distance diagrams taken from the running difference movies. The dynamic up-flows are clearly seen. The position of the crosscuts are shown in Fig. 1 and the same cross-cut positions are used for the left and right columns. 


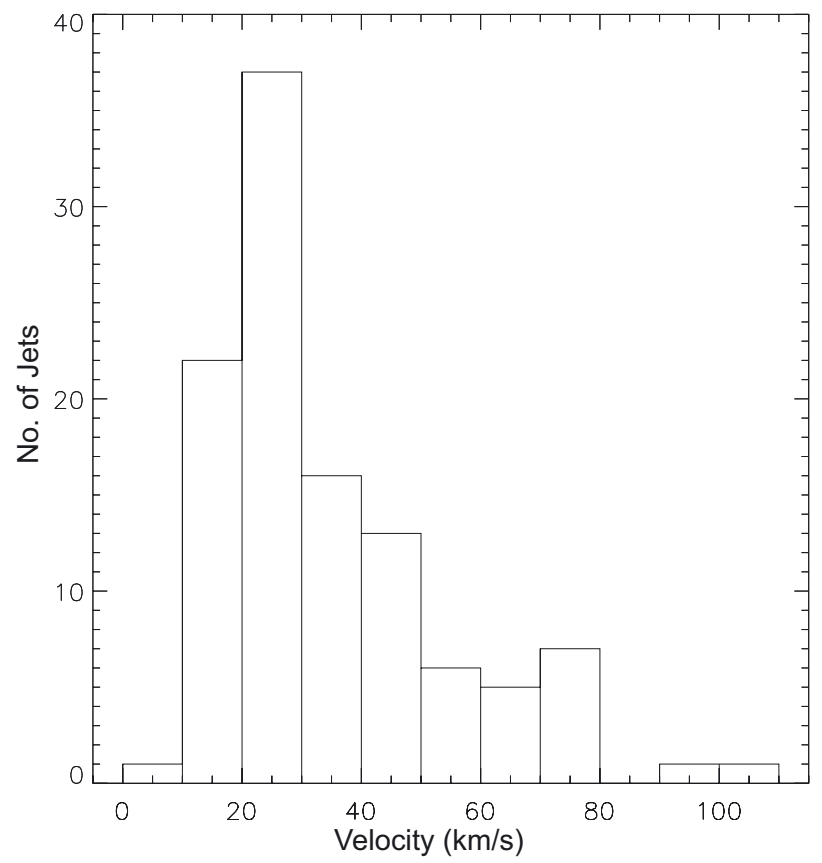

Fig. 4. Histogram of jet velocities calculated from time-distance diagrams. The velocities have a median value of $28 \pm 19 \mathrm{~km} \mathrm{~s}^{-1}$ and are binned in groups of $10 \mathrm{~km} \mathrm{~s}^{-1}$.

jets have a range of upward velocities, $10-110 \mathrm{~km} \mathrm{~s}^{-1}$, with a median value of $28 \pm 19 \mathrm{~km} \mathrm{~s}^{-1}$. The jet velocities have similar values to those reported previously for the type-I spicules, i.e., $10-40 \mathrm{~km} \mathrm{~s}^{-1}$ and also decelerate with height. The deceleration of the jets is readily identified by the curved tracks seen within Fig. 3. The deceleration is around $0.03-0.23 \mathrm{~km} \mathrm{~s}^{-1}$, much less than the expected gravitational deceleration. Similar behavior is observed for spicules. However, the jet plasma is rarely seen to return to the surface, fading from the filter at the jet's peak height.

On the right hand side of the sunspot, we see a similar picture. However, more jets with velocities $>40 \mathrm{~km} \mathrm{~s}^{-1}$ are observed than compared to the left hand side. The high velocity jets are observed typically when large-scale ejections of plasma occur, with numerous jets driven simultaneously over a $6000 \mathrm{~km} \mathrm{spa-}$ tial scale. This large-scale jet excitation only occurs on this side of the sunspot and demonstrates that the driver of these events is relatively localised (possibly connected with the overlying coronal loops).

A number of receding jets are seen and down flows are also observed. The number of these events that we can identify is relatively small, namely 11 events in the $45 \mathrm{~min}$ time series. The events have an average velocity of $12 \pm 8 \mathrm{~km} \mathrm{~s}^{-1}$. This velocity is comparable to down flows seen in TR spectral lines (e.g., Marsch et al. 2004; Dammasch et al. 2008; Teriaca et al. 2008).

One of the most interesting features is, unlike spicules, these jets do not taper with height. It has been often reported that spicule intensity decreases with height (e.g., Beckers 1968) and they have a tapered appearance. In Figs. 1, 2 and 5 it can be observed that the jets have increased emission along the leading edge of the groups, suggesting an increase in temperature or density (or both). The increase in emission at the leading edge is $\sim 5 \%$ of the emission of the the trailing plasma. The enhancement is much clearer in the unsharp masked image (Fig. 5). The fact that only the leading edge is enhanced suggests this is not a line of sight effect, i.e., a superposition jets. If this were the

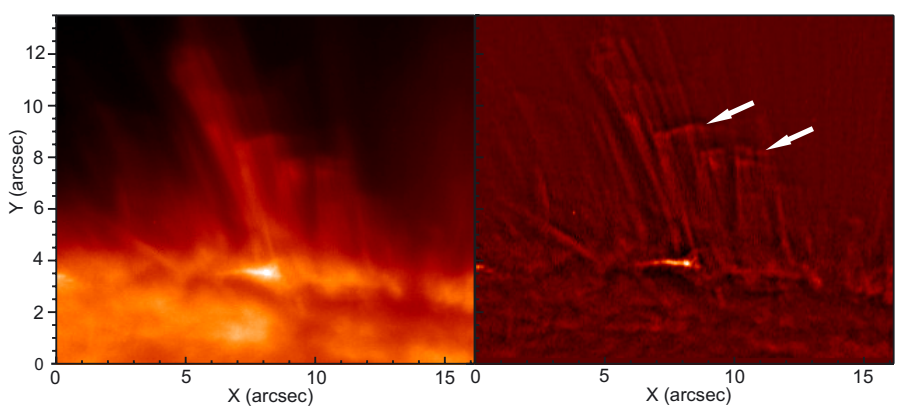

Fig. 5. The left column is a Ca II image. The right column shows an unsharp masked image of the same region. The white arrows mark the enhanced emission at the leading edges of the jet.

case, we would expect most or all of the jet to show enhanced emission, not just the leading edge.

We suggest the cause of this enhanced emission is due to the leading edge of the jet being a shock front. The shock would heat and condense the plasma leading to enhanced emission. If sufficient heating were to occur, then the ionization of $\mathrm{Ca}^{+}$ions would lead to plasma disappearing from the SOT filter. This would explain why some of the ejected plasma is not seen to return to the surface and the lower emission of the returning plasma. However, the unshocked plasma in front of the jet has to have a temperature below $1-2 \times 10^{5} \mathrm{~K}$. This is because the sound speed in the unshocked plasma has to be less than the velocity of the jet $\left(30-40 \mathrm{~km} \mathrm{~s}^{-1}\right)$ or else a shock cannot form. Models of coronal loops suggest that relatively cool plasma, i.e. $T<0.5 \mathrm{MK}$, exists in the loop's legs even at coronal heights (e.g., Aschwanden et al. 2001). The shock heating of chromospheric jets is discussed in, e.g., Hollweg (1982); Sterling \& Hollweg (1988); Sterling (2000); James \& Erdélyi (2002); James et al. (2003); Murawski et al. (2011).

On viewing the TRACE $1550 \AA$ and $1600 \AA$ images (Fig. 1), the observed emission shows similar geometrical features and can be seen to be co-spatial with the $\mathrm{Ca}$ II $\mathrm{H}$ emission seen with SOT. The observed emission above the limb in the TRACE bandpasses is most likely due to the contribution from C IV, which is formed at $T \sim 0.1 \mathrm{MK}$. This would add weight to the suggestion that shock fronts can form at the leading edge of the jet and low level heating is occurring of the chromospheric jet plasma to at least lower to mid TR temperatures.

Unfortunately, the corresponding XRT, TRACE and SOHO/EIT data does not provide sufficient temporal coverage to examine whether the jets observed in $\mathrm{Ca}$ II $\mathrm{H}$ have counterparts at higher temperatures. Other instruments, e.g. SUMER, CDS, also do not have data on this region. Such joint observations are undoubtedly needed to confirm whether the observed jets have higher temperature counterparts and confirm whether shock-waves (or other mechanisms) are heating the chromospheric plasma.

\section{Excitation of the jets}

Now that we have described some of the observational features of the jets, we demonstrate evidence that suggests potential excitation mechanisms. In the quiet Sun it had been suggested that a mixture of p-modes and granular buffeting are the main mechanisms responsible for type-I spicule excitation (De Pontieu et al. 2004; Erdélyi et al. 2007), while reconnection is thought responsible for driving type-II events (De Pontieu et al. 2007b). We give 
evidence that both methods are also at work in the formation of jet events at the edge of sunspots.

\subsection{Reconnection in the sunspot}

Possible sites of magnetic reconnection can be identified in the images. In particular from $t=400 \mathrm{~s}$ to $t=1100 \mathrm{~s}$ after the observations begin, jet excitation appears to be associated with a sustained bright region at the chromospheric level, $\sim 3000 \mathrm{~km}$ in length. In Fig. 6 we display a series of images that portray the typical behavior in the bright region. In the first image a short loop-like structure can be seen. In the next few images, a portion of the loop is seen to rise upward until it protrudes above the low chromosphere and is aligned with existing structures that support up-flowing plasma. This process is seen to occur every $\sim 50$ s while the bright region exists, suggesting multiple reconnection events. The observed event is similar in appearance to reconnecting chromospheric loops seen in large jet events in the quiet chromosphere, e.g., Liu et al. (2009, 2011), Morton et al. (2012a). The jets also display a transverse motion across the sunspot, giving the appearance of the field lines being dragged from right to left. This could be a sign of the magnetic field reorganising above the reconnection site.

\subsection{Timescales of jet excitation}

Now, we investigate the typical timescales associated with the jets. Using the time-distance diagrams shown in Fig. 3, we apply Fourier wavelet analysis at different heights. We find the predominance of a $300 \mathrm{~s}$ timescale at the base of the cross-cuts, both on the left and right hand sides of the sunspot. The presence of timescales with periods of 400-500 s are also significant but have much less power than the $300 \mathrm{~s}$ timescales. The power in the $300 \mathrm{~s}$ timescales drops off rapidly within the first $2 \mathrm{Mm}$ of the cross-cuts. Higher in the atmosphere, the intensity is dominated by $400-500 \mathrm{~s}$ timescales. This timescale appears related to the production of the jets that shoot material into the lower corona (heights in excess of $6 \mathrm{Mm}$ ). In Fig. 3 we have over plotted typical results from the wavelet. The wavelet scale with the greatest power at a particular height is plotted. The shown wavelet scale is plotted so that the zero-crossings correspond to the height at which the wavelet is obtained.

We cannot rule out the inclusion of some plage region fibrils in these cross-cuts contributing to the timescales. Fibrils are seen in the data and a number occur near the sunspot. The fibrils are expected to be a mixture of vertical and inclined structures, however, previous observations (De Pontieu et al. 2007a) suggest both would not reach as high into the corona ( 1-4 Mm) as the sunspot jets. Hence, the fibrils should mainly be observed at the foot points of the sunspot jets, i.e., at the bottom of the cross-cuts. The inclusion of dynamic fibrils could be a reason why $300 \mathrm{~s}$ periods show significant power at the base of the cross-cuts.

The long timescales found here are similar to the periods of waves reported in sunspots, e.g., Thomas et al. (1982), O'Shea et al. (2001), and in coronal loops in active regions, De Moortel et al. (2002). It is possible that waves could be exciting the jets either by non-linear steepening to form shocks, which elevate the chromospheric material (e.g., Hollweg 1982; De Pontieu et al. 2004; Erdélyi et al. 2007) or exciting periodic reconnection (e.g., Heggland et al. 2009; McLaughlin et al. 2010; McLaughlin et al. 2012).

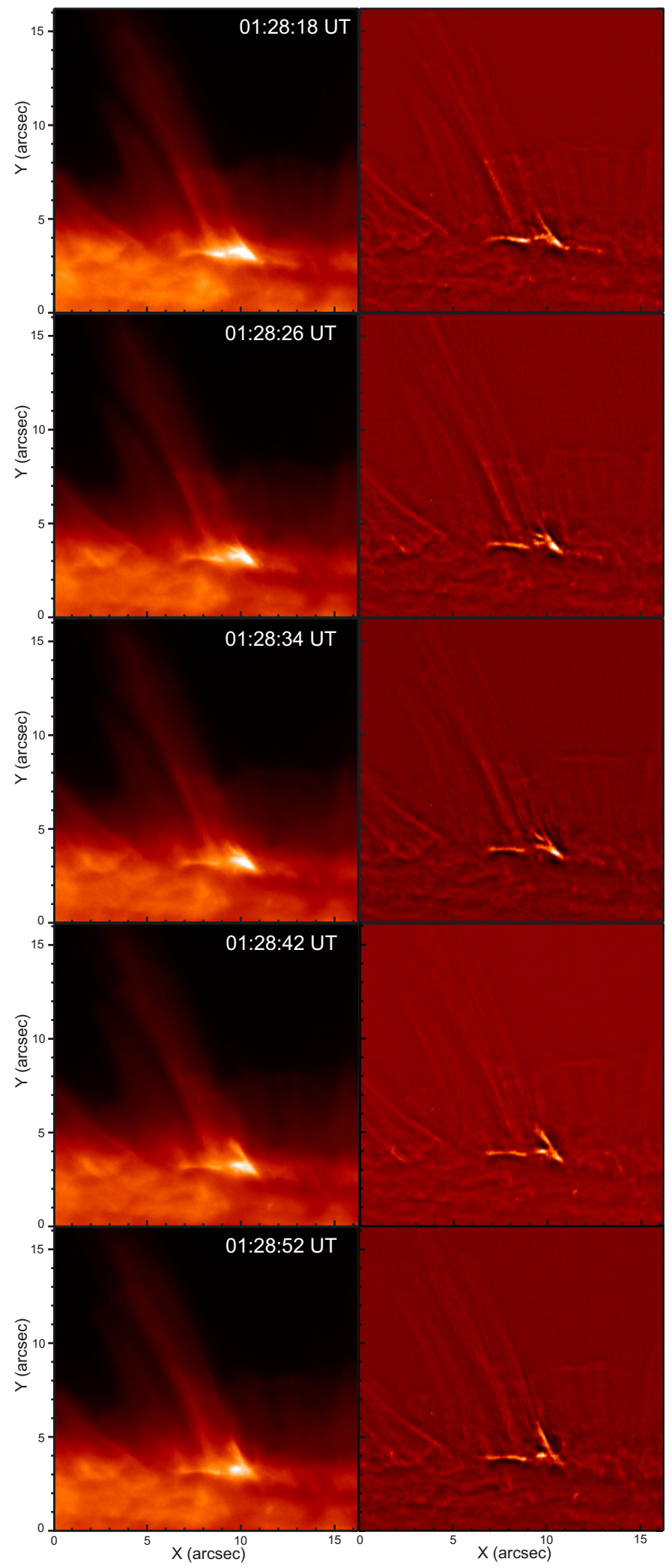

Fig. 6. The left column is Ca II images of a sunspot showing plasma jets and the site of potential magnetic reconnection. Each image is separated in time by $\sim 8 \mathrm{~s}$. The right column shows the unsharp masked images.

We also note the $50 \mathrm{~s}$ timescale associated with the discussed reconnection event does not show significant power (i.e., $>99 \%$ significance) in the wavelet time-series. It would appear the longer timescales (300-500 s) are associated with more 


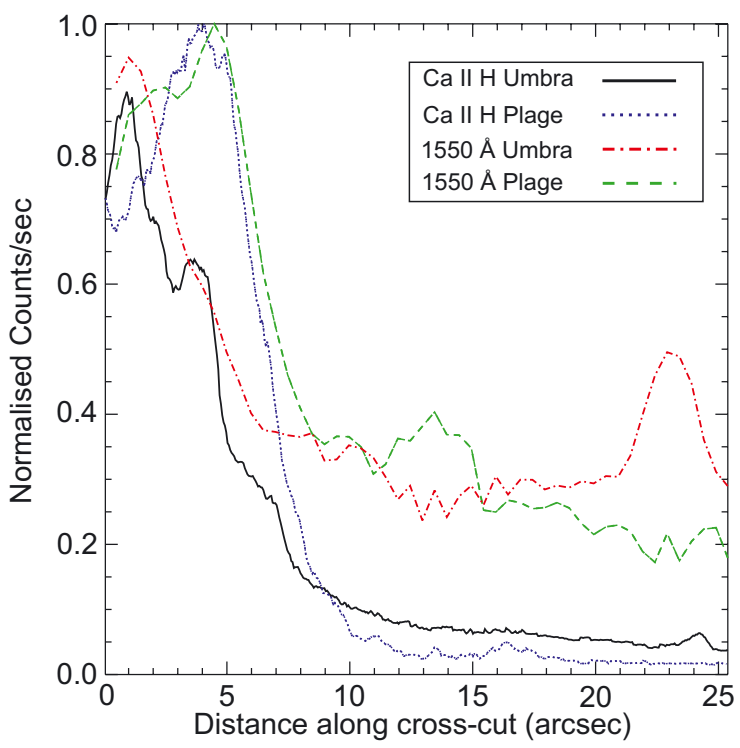

Fig. 7. The flux profiles taken above plage and umbral regions. The $1550 \AA$ and Ca II H plage cross-cuts are the dashed (green) and dotted (blue) lines, respectively. The $1550 \AA$ and Ca II H umbral cross-cuts are the dash-dot (red) and solid (black) lines, respectively. The intensities have been normalised to the largest counts/sec value in the umbral and plage cross-cuts for each filter. The reduced emission in the Ca II H umbral cross-cut at $\sim 1^{\prime \prime}$ along the slit is due to the Wilson depression of the sunspot.

powerful excitation events, able to launch plasma to greater heights above the sunspot. However, the observation of jet excitation on shorter timescales shows that localised events with a range of timescales will also play a significant role in determining the observed dynamics.

\section{The chromosphere and TR above the sunspot}

Flows in sunspot and active region TRs have been studied in detail using spectroscopy (e.g., Kjeldseth-Moe et al. 1988; Marsch et al. 2004; Warren et al. 2011). In particular, Tian et al. (2009) studied the profile of Hydrogen Lyman lines above both plage and active regions. Typically, the Lyman- $\beta$ to Lyman- $\epsilon$ show self-reversal over plage regions but not over the sunspot umbra, suggesting a possible different pattern of flow in the two regions. The differing magnetic structures in each region probably plays a role in this phenomenon.

Tian et al. (2009) suggest that opacity over the sunspot is much less than over the plage, leading to reduced absorption of the Lyman emission on-disk. This in turn implied less chromospheric plasma over sunspots. Further, they calculated electron densities over the plage and umbra from a range of density sensitive lines pairs, finding lower TR densities over the umbra when compared to the plage region. It was suggested this was due to a higher and extend TR over the sunspot in comparison to the plage TR.

We can confirm this conjecture is indeed true. In the Hinode/SOT Ca II H and the TRACE $1550 \AA$ images we have over plotted two solid lines (Fig. 1). The Ca II H cross-cuts are co-spatial with the TRACE cross-cuts. The upper line marks the surrounding plage region and the lower line marks the umbral region of the sunspot. The flux along these cross-cuts is plotted in Fig. 7 for the first image in the $\mathrm{Ca}$ II $\mathrm{H}$ time series and the closest TRACE $1550 \AA$ image in time.
First, we can identify approximately where the solar surface is in the flux profiles. The plage profiles in Fig. 7 show a steep decrease in the flux after $\sim 4$ " along the cross-cut signifying the reduction in photospheric contributions to the observed emission. The umbral $\mathrm{Ca}$ II $\mathrm{H}$ flux also has a sharp decrease at $1^{\prime \prime}$ along the cross-cut before increasing again. This is due to the Wilson depression that can be observed in Fig. 1. The $1550 \AA$ flux also begins to decrease at $1^{\prime \prime}$ implying the $1550 \AA$ emission is also sensitive to the Wilson effect.

Above the solar surface, the TR emission is seen to be significantly greater above the plage than the umbra to a distance of $\sim 9^{\prime \prime}$ along the cross-cut. This suggests the umbral TR is less dense than the plage TR. After $\sim 9^{\prime \prime}$, the emission is then comparable until $\sim 15^{\prime \prime}$ along the cross-cut when the plage TR emission becomes less than the umbral TR emission. Hence, there is more TR temperature material at greater heights above the umbra than the plage. This demonstrates the higher and extended nature of the umbral TR.

In Fig. 7 we also show the chromospheric $\mathrm{Ca}$ II $\mathrm{H}$ emission along taken along the cross-cuts shown in Fig. 1. The chromospheric plage emission is greater than the umbral emission until $9^{\prime \prime}$ along the cross-cut, suggesting a denser chromosphere in the plage. However, the umbral chromosphere can be seen to extend much higher than the plage region, with greater umbral emission after $9^{\prime \prime}$ along the cross-cut. This should be expected considering that the sunspot jets typically reach greater heights than jets reported in the quiet Sun and plage regions.

The attributes of the umbral chromosphere and TR, i.e. lessdense, higher and extended compared to the plage, can probably be put down to the influence of the chromospheric jets. The sunspot jets appear to be more dynamic than previously reported quiet Sun and plage region jets. The jets observed here are able to distribute the chromosphere much higher into the corona, also leaving the chromospheric material thinner above the umbra. In turn this causes the TR to be extended over greater heights above the umbra. This will be the case whether the jets are responsible for generating the TR, e.g., via shock heating, or simply just driving the TR material to a greater height.

\section{Discussion and conclusion}

The jets observed around the edge of the sunspot display much greater dynamism than jets observed previously in the quiet Sun and plage regions. New jets appear almost continuously in groups, ejecting large amounts of plasma along the field lines. Enhanced emission at the leading edge of the jets and the lack of visible down flowing plasma suggests that the jet material may be being heated by shocks as it rises into the sunspot TR/corona. This claim is supported by the presence of TR temperature material (C IV at $T \sim 0.1 \mathrm{MK}$ ) in TRACE $1550 \AA$ and $1600 \AA$ filters that is co-spatial with and shares similar geometric features to the $\mathrm{Ca}$ II $\mathrm{H}$ emission. The shock wave-heating may not provide the hottest plasma observed in active regions, i.e., temperatures in excess of $1 \mathrm{MK}$, but could provide a steady stream of warm plasma $T \sim 0.1 \mathrm{MK}$ at the footpoints of the active regions. The heated jet plasma may add to some of the observed transition region down flows above sunspots (e.g., Kjeldseth-Moe et al. 1988; Brynildsen et al. 1999; Teriaca et al. 2008; Tian et al. 2009) if the heated plasma returns to the surface. However, the TR plasma could be heated further, possibly to coronal temperatures, before it returns to the surface (as suggested in Pneuman \& Kopp 1978). Further studies will have to focus on tracking the jet plasma as it heated to determine its fate. This will require 
both high spatial and temporal resolution observations covering a wide range of temperatures.

We can obtain an estimate for the amount of chromospheric jet plasma that needs to be heated to supply the TR down flows. The technique used is similar to that in, e.g. Athay \& Holzer (1982), De Pontieu et al. (2009).

The mass flux of chromospheric plasma is given by $F_{s}=\epsilon \alpha_{s} n_{s} v_{s}$, where $\epsilon$ is the proportion of mass heated to TR temperatures, $\alpha_{s}$ is the volume filled by the jets, $n_{s}$ is the electron particle density and $v_{s}$ is the up-flow speed of the chromospheric jets. The amount of chromospheric material heated to TR temperatures has to at least equal the downward mass flux seen in the TR lines, i.e., $F_{\mathrm{TR}}=\alpha_{\mathrm{TR}} n_{\mathrm{TR}} v_{\mathrm{TR}}$. The electron density in the TR above sunspots is $\sim 10^{10} \mathrm{~cm}^{-3}$ (Tian et al. 2009), with typical down flow velocities of $10-30 \mathrm{~km} \mathrm{~s}^{-1}$ (Marsch et al. 2004; Dammasch et al. 2008), which gives a mass flux of $F_{\mathrm{TR}}=\alpha_{\mathrm{TR}} 2 \pm 1 \times 10^{16} \mathrm{~cm}^{-2} \mathrm{~s}^{-1}$.

We have demonstrated that the chromosphere above the sunspot umbra is more extended and possibly less-dense than the plage chromosphere. Because the emission measure $E M \propto n^{2}$, we can provide a crude estimate for the umbral chromospheric electron density. Assuming that temperatures and volumes are equal, the ratio of emission in the plage to the umbra gives $E M_{\mathrm{p}} / E M_{\mathrm{U}}=\left(n_{\mathrm{p}} / n_{\mathrm{u}}\right)^{2}$. For the Ca II emission we find $E M_{\mathrm{p}} / E M_{\mathrm{U}}=2.1 \pm 0.5$ for heights between $1-2 \mathrm{Mm}\left(1.3-3^{\prime \prime}\right)$ above the limb. The electron density of jets in plage regions is $n_{\mathrm{p}} \sim 4 \times 10^{11} \mathrm{~cm}^{-3}$ (Beckers 1972), hence $n_{\mathrm{u}} \sim 2.5 \times 10^{11} \mathrm{~cm}^{-3}$. Now $n_{\mathrm{u}}=n_{s}$, hence the mass flux associated with the chromospheric jets is $F_{s}=\epsilon \alpha_{s} 7.5 \times 10^{17} \mathrm{~cm}^{-2} \mathrm{~s}^{-1}$, where $v_{s} \sim 30 \mathrm{~km} \mathrm{~s}^{-1}$. If we assume that the up-flowing/down flowing plasma is confined to individual field lines, then the ratio $\alpha_{\mathrm{TR}} / \alpha_{s}$ will be a measure of the expansion of the magnetic field with height. Estimated field strengths and changes in magnetic field strength with height (Solanki 2003) give $\alpha_{\mathrm{TR}} / \alpha_{s} \sim 2-3$. This gives $\epsilon \sim 8 \pm 4 \%$, so the heating of only a fraction of the chromospheric jets will replenish the mass lost from TR down flows.

Downflows are also observed in the Ca II H data. However, these are the result of jet material returning to the surface under the influence of gravity. No down flows are observed that cannot be connected with the return of a jet.

The data appears to demonstrate that a number of different mechanisms are in action to drive the jets. Large ejections of plasma, distinct magnetic topology and apparent re-organisation of the magnetic field are observed suggesting magnetic reconnection plays a role. The presence of timescales of $300 \mathrm{~s}$ and $500 \mathrm{~s}$ also suggest that waves may play a role in excitation of the jets, whether this is the formation of shocks to drive material upward (e.g., Hollweg 1982; De Pontieu et al. 2004) or driving periodic reconnection (Heggland et al. 2009; McLaughlin et al. 2010; McLaughlin et al. 2012).

The evidence for jet events similar to type-II spicules is limited in these observations. We see a few fast moving events with speeds $40-100 \mathrm{~km} \mathrm{~s}^{-1}$ that fall into the bracket of type-II. The high-speed jets are only visible when a large ejection event occurs, launching plasma up to $14 \mathrm{Mm}$ in to the sunspot corona.

The reported velocities for up-flows in higher temperature lines consistently exceeds $100 \mathrm{~km} \mathrm{~s}^{-1}$, e.g., Tian et al. (2011a). This apparent discrepancy between our results might be able to be explained in a number of ways. In the observations presented here, the lack of evidence for events with rise-speeds in excess of $100 \mathrm{~km} \mathrm{~s}^{-1}$ could be due to the observed transverse motions obscuring any potential signals in time-distance plots. Certain jets move transversally across the sunspot and pass through the cross-cuts leaving an almost straight line in the time-distance plots. We neglected any lines that gave velocities in excess of $125 \mathrm{~km} \mathrm{~s}^{-1}$ as a precaution. The shear volume of jets at the foot point also provides a significant restriction to observing type-II like events due to the superposition of many jets. Very fast type-II events, i.e., with velocities in excess of $100 \mathrm{~km} \mathrm{~s}^{-1}$, may only exist in $\mathrm{Ca}$ II $\mathrm{H}$ for a short period of time before significant plasma heating occurs. In such a scenario, their signal will be hidden amongst the forest of jets observed near the cross-cuts footpoints. Another option is that heated jets which fade from the $\mathrm{Ca}$ II $\mathrm{H}$ filters are accelerated and the rise velocity increases with increasing temperature.

In conclusion, we demonstrate that relatively slow moving jets $\left(v \sim 30 \mathrm{~km} \mathrm{~s}^{-1}\right)$ appear to experience shock heating. While study of the fast moving $\left(v>40 \mathrm{~km} \mathrm{~s}^{-1}\right)$ jet events has demonstrated that the heating of plasma to coronal temperatures may be associated with chromospheric phenomena (e.g., De Pontieu et al. 2011), the role that "slower" jet events could play in generating heated atmospheric plasma should not be overlooked. It may be this role is restricted to low level heating of the plasma up to transition region temperatures. However, even such a low level process could "reduce the load" put upon type-II events (or other heating mechanisms) in supplying heated material to the transition region and corona of active regions.

Acknowledgements. The author thanks M. Ruderman, V. Fedun, G. Verth, A. Hillier, D. Jess and R. Erdélyi for useful and insightful discussions. Thanks goes to the Science and Technology Facilities Council (STFC), UK for the financial support received. The analysis has been based on observations done by the Hinode satellite. Hinode is a Japanese mission developed and launched by ISAS/JAXA, with NAOJ as domestic partner and NASA and STFC (UK) as international paertners. Is it operated by these agencies in co-operation with ESA and NSC (Norway).

\section{References}

Aschwanden, M. J., Schrijver, C. J., \& Alexander, D. 2001, ApJ, 550, 1036 Athay, R. G., \& Holzer, T. E. 1982, ApJ, 255, 743

Beckers, J. M. 1968, Sol. Phys., 3, 367

Beckers, J. M. 1972, ARA\&A, 10, 73

Bryans, P., Young, P. R., \& Doschek, G. A. 2010, ApJ, 715, 1012

Brynildsen, N., Maltby, P., Brekke, P., Haugan, S. V. H., \& Kjeldseth-Moe, O. 1999, Sol. Phys., 186, 141

Cirtain, J. W., Golub, L., Lundquist, L., et al. 2007, Science, 318, 1580

Dammasch, I. E., Curdt, W., Dwivedi, B. N., \& Parenti, S. 2008, Annales Geophysicae, 26, 2955

De Moortel, I., Ireland, J., Hood, A. W., \& Walsh, R. W. 2002, A\&A, 387, L13

De Pontieu, B., Erdélyi, R., \& de Wijn, A. G. 2003, ApJ, 595, L63

De Pontieu, B., Erdélyi, R., \& James, S. P. 2004, Nature, 430, 536

De Pontieu, B., Hansteen, V. H., Rouppe van der Voort, L., van Noort, M., \& Carlsson, M. 2007a, ApJ, 655, 624

De Pontieu, B., McIntosh, S., Hansteen, V. H., et al. 2007b, PASJ, 59, 655

De Pontieu, B., McIntosh, S. W., Carlsson, M., et al. 2007c, Science, 318, 1574

De Pontieu, B., McIntosh, S. W., Hansteen, V. H., \& Schrijver, C. J. 2009, ApJ, 701, L1

De Pontieu, B., McIntosh, S. W., Carlsson, M., et al. 2011, Science, 331, 55

Delaboudinière, J.-P., Artzner, G. E., Brunaud, J., et al. 1995, Sol. Phys., 162, 291

Erdélyi, R., Malins, C., Tóth, G., \& de Pontieu, B. 2007, A\&A, 467, 1299

Handy, B. N., Bruner, M. E., Tarbell, T. D., et al. 1998, Sol. Phys., 183, 29

Handy, B. N., Acton, L. W., Kankelborg, C. C., et al. 1999, Sol. Phys., 187, 229

Heggland, L., De Pontieu, B., \& Hansteen, V. H. 2009, ApJ, 702, 1

Hollweg, J. V. 1982, ApJ, 257, 345

James, S. P., \& Erdélyi, R. 2002, A\&A, 393, L11

James, S. P., Erdélyi, R., \& De Pontieu, B. 2003, A\&A, 406, 715

Kamio, S., Peter, H., Curdt, W., \& Solanki, S. K. 2011, A\&A, 532, A96

Kano, R., Sakao, T., Hara, H., et al. 2008, Sol. Phys., 249, 263

Katsukawa, Y., Berger, T. E., Ichimoto, K., et al. 2007, Science, 318, 1594

Kjeldseth-Moe, O., Brynildsen, N., Brekke, P., et al. 1988, ApJ, 334, 1066

Kuridze, D., Morton, R. J., Erdélyi, R., et al. 2012, ApJ, 750, 51

Liu, W., Berger, T. E., Title, A. M., \& Tarbell, T. D. 2009, ApJ, 707, L37

Liu, W., Berger, T. E., Title, A. M., Tarbell, T. D., \& Low, B. C. 2011, ApJ, 728,

103 


\section{R. J. Morton: Chromospheric jets}

Madjarska, M. S., Vanninathan, K., \& Doyle, J. G. 2011, A\&A, 532, L1 Marsch, E., Wiegelmann, T., \& Xia, L. D. 2004, A\&A, 428, 629

Marsch, E., Tian, H., Sun, J., Curdt, W., \& Wiegelmann, T. 2008, ApJ, 685, 1262

McLaughlin, J. A., Hood, A. W., \& de Moortel, I. 2010, Space Sci. Rev., 62

McLaughlin, J. A., Verth, G., Fedun, V., \& Erdélyi, R. 2012, ApJ, 749, 30

Moore, R. L., Sterling, A. C., Cirtain, J. W., \& Falconer, D. A. 2011, ApJ, 731, L18

Morton, R. J., Srivastava, A. K., \& Erdélyi, R. 2012a, A\&A, 542, A70

Morton, R. J., Verth, G., McLaughlin, J. A., \& Erdélyi, R. 2012b, ApJ, 744, 5

Murawski, K., Srivastava, A. K., \& Zaqarashvili, T. V. 2011, A\&A, 535, A58

O'Shea, E., Banerjee, D., Doyle, J. G., Fleck, B., \& Murtagh, F. 2001, A\&A, 368, 1095

Peter, H. 2010, A\&A, 521, A51

Pneuman, G. W., \& Kopp, R. A. 1978, Sol. Phys., 57, 49

Rouppe van der Voort, L., Leenaarts, J., de Pontieu, B., Carlsson, M., \& Vissers, G. 2009, ApJ, 705, 272
Scullion, E., Erdélyi, R., Fedun, V., \& Doyle, J. G. 2011, ApJ, 743, 14 Solanki, S. K. 2003, A\&ARv, 11, 153

Sterling, A. C. 2000, Sol. Phys., 196, 79

Sterling, A. C., \& Hollweg, J. V. 1988, ApJ, 327, 950

Suematsu, Y., Tsuneta, S., Ichimoto, K., et al. 2008, Sol. Phys., 249, 197

Teriaca, L., Curdt, W., \& Solanki, S. K. 2008, A\&A, 491, L5

Thomas, J. H., Cram, L. E., \& Nye, A. H. 1982, Nature, 297, 485

Tian, H., Curdt, W., Teriaca, L., Landi, E., \& Marsch, E. 2009, A\&A, 505, 307

Tian, H., McIntosh, S. W., \& De Pontieu, B. 2011a, ApJ, 727, L37

Tian, H., McIntosh, S. W., Rifal Habbal, S., \& He, J. 2011b, ApJ, 736, 130

Tziotziou, K., Tsiropoula, G., \& Mein, P. 2004, A\&A, 423, 1133

Ugarte-Urra, I., \& Warren, H. P. 2011, ApJ, 730, 37

Vasheghani Farahani, S., Van Doorsselaere, T., Verwichte, E., \& Nakariakov, V. M. 2009, A\&A, 498, L29

Warren, H. P., Ugarte-Urra, I., Young, P. R., \& Stenborg, G. 2011, ApJ, 727, 58

Zaqarashvili, T. V., \& Erdélyi, R. 2009, Space Sci. Rev., 149, 355 